\title{
Relocation of Active Acetylcholinesterase to Liposome-Gel Conjugate
}

\author{
Yukihisa Okumura $^{\mathrm{a}, *}$, Hiroki Mizushima ${ }^{\mathrm{b}}$ and Junzo Sunamoto ${ }^{\mathrm{b}, \dagger}$ \\ ${ }^{a}$ Department of Chemistry and Material Engineering, Faculty of Engineering, \\ Shinshu University, 4-17-1 Wakasato, Nagano 380-8553, Japan \\ ${ }^{\mathrm{b}}$ Department of Synthetic Chemistry and Biological Chemistry, Graduate School of \\ Engineering, Kyoto University, Yoshida-Hommachi, Sakyo, Kyoto, 606-8501, Japan
}

*Corresponding author. Tel.: +26-269-5399; fax: +26-269-5424.

E-mail address: okumura@shinshu-u.ac.jp (Y. Okumura).

${ }^{\dagger}$ Deceased on December 6, 2005. 
Abstract

Relocation of a glycosylphosphatidylinositol (GPI) anchored protein acetylcholinesterase (AChE) in its enzymatically active form from proteovesicles containing human erythrocyte ghost membrane proteins onto a liposome-gel conjugate was examined. Liposomes of 1,2-dimyristoylphosphatidylcholine (DMPC) were immobilized on Sephacryl S-1000 gel that was chemically modified to bear hydrophobic octyl moieties. Upon coincubation of the liposome-gel conjugate with freely suspended proteovesicles prepared from erythrocyte ghosts, $50 \%$ of the AChE left the proteovesicles and immobilized onto the liposome-gel conjugate in 18 hours. When the proteovesicles were immobilized and interacted with freely suspended plain liposomes, approximately $2 \%$ of the AChE appeared in the liposome fraction. The relocation of AChE apparently possesses strong preference for the liposome-gel conjugate, suggesting that the hydrophobic moieties on the gel could assist the relocation.

Keywords: Liposome; Proteovesicle; Acetylcholinesterase; Liposome immobilization; Protein transfer; Glycosylphosphatidylinositol-anchored protein; Cross-linked polymer gel; Protein painting; Protein reconstitution 
Relocation of glycosylphosphatidylinositol (GPI)-anchored proteins is a key process in a few biochemical/biophysical topics of active interest. One is protein transfer to liposomes, in which coincubation of biological cells with artificial lipid bilayer membrane vesicles (liposomes) results in release of the membrane proteins into the liposomal fraction $[1,2]$. The released proteins were found incorporated in lipid membrane vesicles that bear resemblance to the interacted liposomes, and hence the phenomenon has been described as "transfer" of proteins from cells to liposomes. The process was most studied with human erythrocyte proteins [1-9], and the release of acetylcholinesterase (AChE), a GPI-anchored protein, has been extensively monitored as it may offer a clue to the elucidation of the release mechanism, which has been under discussion [6,7,9].

Recently, modification of the function of a cell by reintegrating an exogenous membrane protein into the plasma membrane of the cell, or "protein painting," was proposed [10,11]. GPI-anchored proteins are found particularly suitable for this technique, and hence the clarification of their reintegration process has become important. Furthermore, relocation of erythrocyte membrane proteins from proteovesicles onto lymphoid cells has been known [4], and this leads to an interesting possibility of protein painting by directly using proteovesicles, which may be also worth further examination.

For better understanding of these mutually related subjects, a study of the relocation of a GPI-anchored protein in a model system is called for. In the present study, a cell-free system was constructed with plain liposomes and AChE-bearing proteovesicles. Either of the two types of the membrane vesicles was immobilized on a hydrophobically modified cross-linked polymer gel, and relocation of AChE in the system was examined.

\section{Materials and methods}

Materials. A human erythrocyte concentrate was kindly donated from Kyoto Red Cross Blood Center. Hydrophobically modified Sephacryl S-1000 gel was prepared according to Yang et al (bearing $0.007 \mathrm{mmol}$ of octyl moieties per $\mathrm{ml}$ of the swelled gel) [12] 1,2-Dimyristoylphosphatidylcholine (DMPC) was purchased from Sigma Aldrich Japan (Tokyo, Japan). Phosphatidylcholine was assayed as choline using a commercially available reagent kit (Phospholipid C-Test Wako, Wako Pure Chemicals, Tokyo, Japan). Protein concentration was determined by using the BCA Protein Assay Reagent (Pierce, 
Rockford, IL).

Preparation of liposomes and proteovesicles. Briefly, a thin film of DMPC (35 $\mathrm{mg}$ ) formed on a flask wall was swelled and suspended in $5.0 \mathrm{ml}$ of a HEPES buffer (10 mM HEPES containing $150 \mathrm{mM} \mathrm{NaCl}, \mathrm{pH}$ 7.4). The crude liposome suspension thus obtained was extruded through a series of polycarbonate membrane filters (from $1000 \mathrm{~nm}$ to $100 \mathrm{~nm}$ ). The average diameter of the liposomes was approximately $100 \mathrm{~nm}$ as determined by dynamic light scattering measurement (Photal DLS-700, Osaka, Japan).

Ghosts were prepared from an erythrocyte concentrate by hemolysis in a hypotonic phosphate buffer (20 mOsm, pH 7.4) and following reequilibration in an isotonic HEPES buffer (10 mM HEPES containing $150 \mathrm{mM} \mathrm{NaCl}, \mathrm{pH}$ 7.4). The ghost suspension (protein concentration $6.0 \mathrm{mg} / \mathrm{ml}$ ) was mixed with an equal volume of a liposome suspension (lipid concentration $10 \mathrm{mM}$ ) and incubated for $180 \mathrm{~min}$ at $37{ }^{\circ} \mathrm{C}$. Removal of the ghost residue by centrifugation $\left(20,000 \mathrm{x} \mathrm{g}, 4{ }^{\circ} \mathrm{C}\right)$ yielded proteovesicles, which contained AChE among other major erythrocyte proteins [6]. Throughout the study, AChE was assayed as its enzymatic activity in hydrolysis of acetylthiocholine [9]. Typically, 10-15\% of the AChE activity initially on the ghosts was found on the proteovesicles.

Immobilization and interaction of vesicles. In Experiment A, liposomes $(6.0 \mathrm{ml}$, lipid concentration $5.0 \mathrm{mM}$ ) were coincubated with modified Sephacryl gel (swelled bed volume $0.50 \mathrm{ml}$ ) at $37{ }^{\circ} \mathrm{C}$. Liposomes left unattached were removed by thoroughly washing the gel with the HEPES buffer. The liposome-gel conjugate thus obtained was then incubated with a proteovesicle suspension ( $5.0 \mathrm{ml}$, lipid concentration $2.0 \mathrm{mM}$ ) at $37^{\circ} \mathrm{C}$. A small amount (0.3-0.5 ml) of the mixture was taken and passed through a membrane filter (pore size $450 \mathrm{~nm}$ ). The AChE activity and the amount of the lipid on the gel and in the filtrate were then determined.

In Experiment B, proteovesicles $(5.0 \mathrm{ml}$, lipid concentration $2.0 \mathrm{mM})$ were immobilized on modified Sephacryl gel $(0.50 \mathrm{ml})$ at $37{ }^{\circ} \mathrm{C}$ for 24 hours. The proteovesicle-gel conjugate was then coincubated with a plain liposome suspension $(5.0 \mathrm{ml}$, lipid concentration $5.0 \mathrm{mM}$ ).

In Experiment C, the liposome-gel conjugate bearing AChE activity obtained in Experiment A was mixed with a plain liposome suspension $(5.0 \mathrm{ml}$, lipid concentration 10.0 $\mathrm{mM}$ ). The mixture was then incubated at $37^{\circ} \mathrm{C}$. 


\section{Results and Discussion}

In Experiment A, plain DMPC liposomes were first immobilized on Sephacryl S-1000 gel that was modified to bear octyl groups [12]. Typically, $0.040 \mathrm{mmol}$ of the liposomal phospholipid was firmly immobilized per ml of the swelled gel. No spontaneous desorption of the phospholipid was detected for four days in a buffer.

The liposome-gel conjugate was then coincubated with freely suspended proteovesicles. Immediately, AChE activity started to disappear from the proteovesicle fraction (Fig. 1). In 18 hours, $63 \%$ of the activity initially on the proteovesicles was lost. Meanwhile, 95\% of the disappeared AChE was found on the conjugate after 30 hours.

During the coincubation, the proteovesicles fraction also lost $15 \%$ of its phospholipids in 18 hours (Fig. 1). This loss of the phospholipid means direct adsorption of the proteovesicles to the liposome-gel conjugate. However, the adsorption could only account for a minor part (15\%) of the apparent AChE activity transfer. The ratio of the AChE activity to the amount of the lipid in the proteovesicle fraction decreased continuously during the coincubation (Fig. 2). This indicates the presence of an AChE transfer process faster than the vesicle adsorption. Approximately 50\% of the AChE is considered to have left the proteovesicle membrane and moved onto the liposome-gel conjugate in 18 hours.

The AChE extracted onto the liposome-gel conjugate was firmly immobilized. No spontaneous release of the AChE activity was seen upon coincubation of the AChE-bearing conjugate with a buffer. Meanwhile, there was slow but noticeable decrease of the AChE activity on the liposome-gel conjugate. The conjugate in a buffer lost 32\% of the AChE activity in 2 days at $37{ }^{\circ} \mathrm{C}$ (for freely suspended proteovesicles, less than 5\%). The loss may be kept at a smaller level if preserved at a lower temperature.

In Experiment B, proteovesicles and plain liposomes switched their places, and extraction of AChE from immobilized proteovesicles with freely suspended plain liposomes was examined. As in the case of plain liposomes, proteovesicles were firmly immobilized on the gel. Spontaneous desorption of neither vesicles nor AChE from the proteovesicle-gel conjugate in a buffer was detected for 4 days. Although a slow gradual loss of the AChE activity was observed, the conjugate retained more than $80 \%$ of the initial AChE activity after 21 hours from the immobilization.

Upon coincubation of the proteovesicle-gel conjugate with DMPC liposomes, 
AChE activity appeared in the free liposome fraction (Fig. 3). However, the extent of the AChE release was far smaller than that in Experiment A. The release reached a plateau at 27 hours when $4 \%$ of the AChE activity initially on the conjugate was found in the liposome fraction.

Considering the extensive relocation of AChE in Experiment $\mathrm{A}$ and the poor extraction in Experiment B, it is clear that AChE preferably stays on a vesicle/liposome-gel conjugate rather than on freely suspended membrane vesicles. This point was further demonstrated in Experiment C, in which the AChE-bearing liposome-gel conjugate obtained in Experiment A was further interacted with fresh DMPC liposomes. The coincubation released $5 \%$ of the AChE to the liposome fraction in 19 hours. The release behavior resembled that in Experiment B, suggesting similarity between the AChE that was relocated onto the liposome-gel conjugate in Experiment A and that on the proteovesicle-gel conjugate in Experiment B.

In all the experiments, the release of AChE started immediately after the beginning of the coincubation. In contrast, membrane protein release from erythrocytes or their ghosts upon interaction with liposomes always accompanies an induction period prior to the release (for example, 1 hour under the conditions in the present study) [7]. The absence of the induction period suggests that the proteovesicles lack certain constraint, which is present in the plasma membrane and preventing its AChE from relocation.

\section{Summary}

The present study found that membrane-incorporated AChE can be taken out from lipid bilayer membrane, however, only with a help of an appropriate agent such as liposome. And the quite low extent of transfer observed in Experiment B indicates that simple liposome alone should not be sufficient for efficient transfer. The slow gradual loss of the AChE activity on AChE-bearing vesicle-gel conjugates suggests the presence of interaction between the enzyme and the hydrophobic alkyl moieties on the gel, which could provide the assistance for the transfer.

As a method in protein reconstitution, this study demonstrated the successful extraction and immobilization of AChE in its enzymatically active form by using a conjugate of liposomes and a hydrophobically modified cross-linked polymer gel. Regarding the 
feasibility of protein painting using proteovesicles, the rather low extent of the AChE relocation from the proteovesicle-gel conjugate to the liposomal fraction observed in Experiment B is somewhat discouraging. However, a part of the AChE, though small, could certainly be transferred out to another lipid bilayer membrane. In the present system, the affinity of AChE for the liposome-gel conjugate may be too high to allow the release of the extracted AChE. Optimization of the affinity, possibly by modification of the hydrophobic moieties on the gel, could result in the reintegration of extracted GPI-anchored proteins with improved efficiency.

\section{Acknowledgements}

The authors are grateful to Kyoto Red Cross Blood Center for the human erythrocyte concentrate. A part of the study was supported by Grant-in-Aids for Scientific Research (B) (16310099) from Japan Society for the Promotion of Sciences (JSPS). 


\section{References}

[1] S.R. Bouma, F.W. Brislane, W.H. Huestis, J. Biol. Chem. 252 (1977) 6759.

[2] Y. Okumura, J. Sunamoto, Supramol. Sci. 3 (1996) 171.

[3] W.H. Huestis, A.C. Newton, J. Biol. Chem. 261 (1986) 16274.

[4] A.C. Newton, W.H. Huestis, Biochemistry 27 (1988) 4655.

[5] S.I. Waters, R. Sen, L.S. Brunauer, W.H. Huestis, Biochemistry 35 (1996) 4002.

[6] K. Suzuki, Y. Okumura, T. Sato, J. Sunamoto, Proc. Japan Acad. 71 (B) (1995) 93.

[7] K. Suzuki, Y. Okumura, J. Sunamoto, J. Biochem. (Tokyo) 125 (1999) 876.

[8] K. Suzuki, Y. Okumura, Arch. Biochem. Biophys. 379 (2000) 344.

[9] K. Suzuki, Y. Okumura, Biochemistry 39 (2000) 9477.

[10] M.E. Medof, S. Nagarajan, M.L. Tykocinski, FASEB J. 10 (1996) 574.

[11] D.R.D. Premkumar, Y. Fukuoka, D. Sevlever, E. Brunschwig, T.L. Rosenberry, M.L. Tykocinski, M.E. Medof, J. Cell. Biochem. 82 (2001) 234.

[12] Q. Yang, M. Wallsten, P. Lundahl, Biochim. Biophys. Acta 938 (1988) 243. 
Figure Captions

Fig. 1. Acetylcholinesterase activity and phospholipid in proteovesicle fraction in Experiment A. Plain DMPC liposomes were immobilized on Sephacryl S-1000 gel that was hydrophobized with octyl moieties, and the DMPC liposome-Sephacryl S-1000 gel conjugate (0.040 mmol DMPC per $\mathrm{ml}$ of the swelled gel) was coincubated with freely suspended proteovesicles bearing human erythrocyte membrane proteins (phospholipid concentration $2.0 \mathrm{mM}$ ) at $37{ }^{\circ} \mathrm{C}$, and acetylcholinesterase (AChE) activity (open) and phospholipid (filled) in proteovesicle fraction were determined. The loss of the lipid indicates direct adsorption of the proteovesicles to the conjugate.

Fig. 2. Ratio of AChE activity to phospholipid in proteovesicle fraction in Experiment A. The decrease in the ratio indicates faster relocation of AChE than that of the lipid from the proteovesicle fraction. The values were calculated from the data in Fig. 1.

Fig. 3. AChE activity in liposome fraction in Experiment B. Proteovesicles bearing human erythrocyte membrane proteins (phospholipid concentration $2.0 \mathrm{mM}$ ) were immobilized on Sephacryl S-1000 gel modified with octyl moieties, and the proteovesicle-gel conjugate was coincubated with freely suspended plain DMPC liposomes (phospholipid concentration $5.0 \mathrm{mM}$ ) at $37^{\circ} \mathrm{C}$. 
Fig. 1

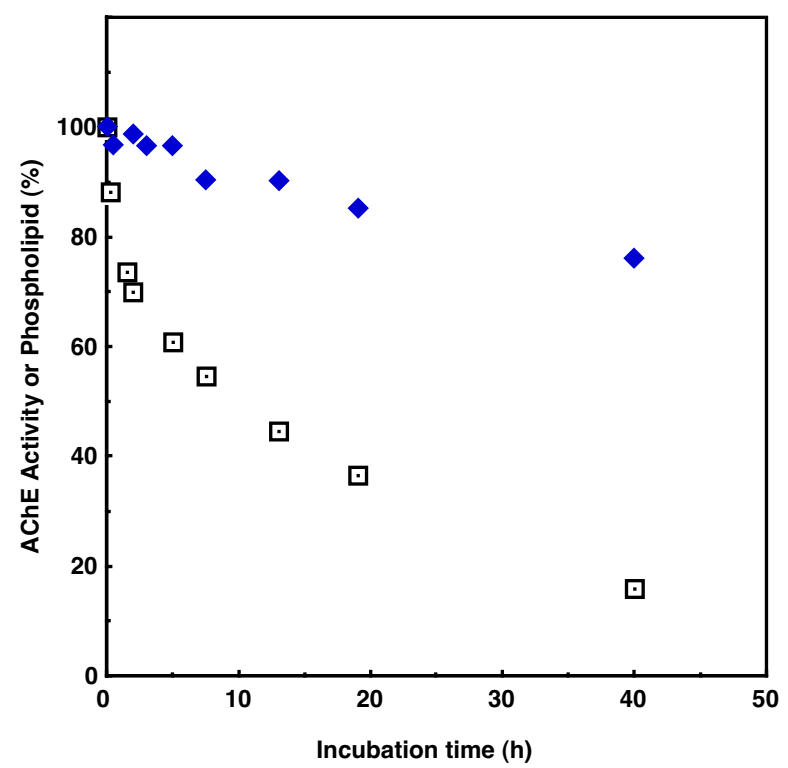

Fig. 2

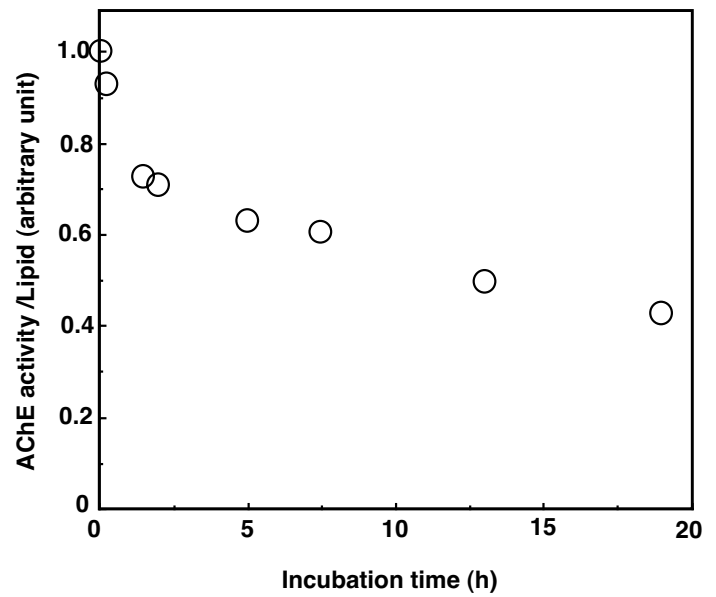


Fig. 3

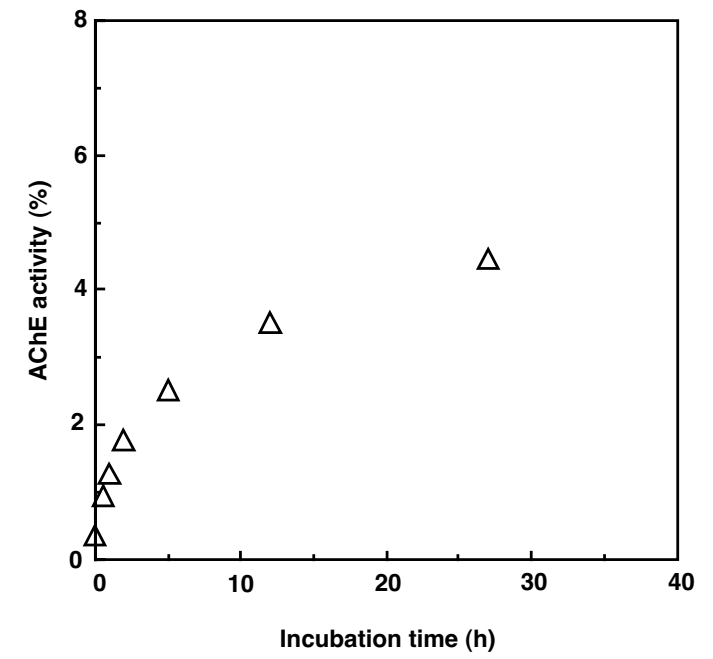

